\title{
The relationship between health-related quality of life and demographic characteristics in patients with chronic hepatitis B
}

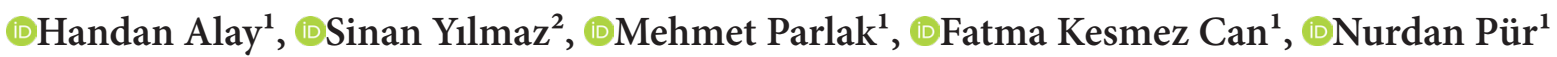 \\ ${ }^{1}$ Atatürk University Faculty of Medicine, Department of Infectious Diseases and Clinical Microbiology, Erzurum, Turkey \\ ${ }^{2}$ Atatürk University Faculty of Medicine, Department of Public Health, Erzurum, Turkey
}

Cite this article as: Alay H, Yllmaz S, Parlak M, Kesmez Can F, Pür N. The relationship between health-related quality of life and demographic characteristics in patients with chronic hepatitis B. Anatolian Curr Med J 2021; 3(2); 130-135.

\begin{abstract}
Introduction: The evaluation of health-related quality of life at specific intervals in chronic hepatitis B (CHB) patients is important in terms of producing interventions aimed at raising quality of life.

Aim: The purpose of this study was to measure health-related quality of life in CHB patients and to evaluate its association with demographic characteristics.

Material and Method: The study was conducted with CHB patients presenting to the infectious diseases clinic between 01 January and 15 March 2020. Date were collected demographic characteristics, and with the short form-36 (SF-36).

Results: Two hundred fourteen patients diagnosed with $\mathrm{CHB}$ and receiving nucleos(t)ide therapy were included in the study. Patients' mean age was $41.9 \pm 12.9$ years, $60.7 \%(n=130)$ were men, $83.6 \%$ were married, and $44.9 \%$ were educated to middle school level. Mean scores on all subscales were higher among men. Patients' SF-36 subscale scores differed significantly in terms of education. Unmarried individuals registered higher mean scales on the SF-36 subscales compared to married participants. The score distributions of housewives and clerical workers differed significantly on the physical functioning (PF), social functioning (SF), and physical role limitation (PRL) subscales $(\mathrm{p}<0.001, \mathrm{p}=0.004$, and $\mathrm{p}=0.003$, respectively). Patients' mental health subscale scores were significantly differently distributed depending on smoking status $(\mathrm{p}=0.015)$. $P F, P R L$, social role limitation, and energy/fatigue subscale scores differed significantly between participants living in urban areas and those from outlying districts or villages $(\mathrm{p}<0.01)$.

Conclusion: It is of great importance for patients' demographic characteristics to be evaluated during follow-up and for appropriate clinical support to be provided when required.
\end{abstract}

Keywords: Chronic hepatitis B, quality of life, demographic characteristics

\section{INTRODUCTION}

Approximately 250 million people worldwide suffer from chronic hepatitis B (CHB) infection (1). CHB patients are at risk of developing end-stage liver disease such as cirrhosis, liver failure, and hepatocellular carcinoma. Antiviral therapy suppresses hepatitis B virus (HBV) replication in these patients, and survival rates rise. Nucleos(t)ide analogues used in treatment are safe and effective antiviral agents recommended in the first stage of treatment in most guidelines. Although these drugs powerfully inhibit $\mathrm{HBV}$ replication, they are unable to eliminate the virus (2-4).

Quality of life refers to the general perception of the individual's positive and negative aspects (5). Health- related quality of life (HRQoL) emerges as an important factor in chronic diseases, and especially CHB. The quality of life of $\mathrm{CHB}$ patients can be adversely affected by lengthy antiviral therapy and follow-up (6). The regular evaluation of HRQoL in CHB patients, even in the absence of advanced liver disease is important in the adoption of measures aimed at improving quality of life. The purpose of this study was to measure HRQoL in patients with $\mathrm{CHB}$ and to evaluate its associations with sociodemographic characteristics. 


\section{MATERIAL AND METHOD}

The study was approved by Atatürk University Faculty of Medicine Ethical Committee (Ethics approval certificate: B.30.2.ATA.0.01.00/95). All procedures were performed adhered to the ethical rules and the Helsinki Declaration of Principles.

The study was performed with $\mathrm{CHB}$ patients presenting to the infectious diseases clinic between 01 January and 15 March 2020. Patients aged over 18, receiving antiviral therapy with diagnoses of $\mathrm{CHB}$, and consenting to take part were included in the study. Patients with cirrhosis and not receiving antiviral therapy were excluded.

Data were collected using a questionnaire developed by the author investigating various demographic characteristics (sex, age, marital status, place of residence, smoking status and/or alcohol use, duration of disease and accompanying diseases) and with the Turkish version of the short form of the quality of life questionnaire (SF36). The questionnaires were completed using the faceto-face interview method.

\section{The Short Form of the Quality of Life Questionnaire (SF-36).}

This was developed in 1989 by Ware and Sherbourne for clinical application and research. The scale consists of 36 items under two main categories, physical and mental health. It consists of a number of subscales-general health, vitality, physical functioning, role physical, bodily pain, mental health, role emotional, and social functioning. Each heading is scored from a minimum of 0 to a maximum of 100 .Higher scores indicate more favorable HRQoL (7).SF-36 was adapted into Turkish by Pınar et al. (8) and Koçyiğit et al. (9).

\section{Statistical Analysis}

Data analysis was performed on Statistical Package for the Social Sciences (SPSS v20) software. Categorical variables were expressed as number and percentage, and numerical variables as mean plus standard deviation. Normality of distribution of variables was evaluated using the Kolmogorov Smirnov test, $\mathrm{z}$ values for skewness and kurtosis, and charts. The Mann Whitney U test was applied to compare non-normally distributed numerical variables between two groups, the Kruskal Wallis test for comparisons between more than two groups, while the Mann Whitney $\mathrm{U}$ test with Bonferroni correction were applied at post-hoc analyses. Relationships between variables were investigated using Spearman's rho correlation analysis. p values $<0.05$ were regarded as significant for all analyses.

\section{RESULTS}

\section{Sociodemographic and Clinical Characteristics}

Two hundred fourteen patients diagnosed with $\mathrm{CHB}$ and receiving nucleos $(\mathrm{t})$ ide therapy were included in the study. The patients' mean age was $41.9 \pm 12.9$ years, $60.7 \%$ $(\mathrm{n}=130)$ were men, $83.6 \%$ were married, and $44.9 \%$ had been educated to middle school level. The mean number of children per participant was3.0 \pm 1.6 . Cases' sociodemographic characteristics are shown in Table 1.

\begin{tabular}{|c|c|c|}
\hline Variables & $\mathbf{n}$ & $\%$ \\
\hline \multicolumn{3}{|l|}{ Sex } \\
\hline Female & 84 & 39.3 \\
\hline Male & 130 & 60.7 \\
\hline \multicolumn{3}{|l|}{ Education level } \\
\hline Primary school & 10 & 4.7 \\
\hline Middle school & 96 & 44.9 \\
\hline High school & 48 & 22.4 \\
\hline University & 60 & 28.0 \\
\hline \multicolumn{3}{|l|}{ Marital status } \\
\hline Married & 179 & 83.6 \\
\hline Single & 35 & 16.4 \\
\hline \multicolumn{3}{|l|}{ Occupation } \\
\hline Unemployed & 38 & 18.0 \\
\hline Manual & 69 & 32.7 \\
\hline Clerical & 33 & 15.6 \\
\hline Housewife & 61 & 28.9 \\
\hline Student & 10 & 4.7 \\
\hline \multicolumn{3}{|l|}{ Smoking status } \\
\hline Non-smoker & 180 & 84.1 \\
\hline Smoker & 34 & 15.9 \\
\hline \multicolumn{3}{|c|}{ Alcohol consumption } \\
\hline No & 210 & 98.1 \\
\hline Yes & 4 & 1.9 \\
\hline \multicolumn{3}{|l|}{ Place of residence } \\
\hline Urban area & 148 & 69.2 \\
\hline District & 53 & 24.8 \\
\hline Village & 13 & 6.1 \\
\hline \multicolumn{3}{|c|}{ Additional disease } \\
\hline No & 192 & 89.7 \\
\hline Yes & 22 & 10.3 \\
\hline
\end{tabular}

The mean duration of disease was $102.7 \pm 104.0$ months, and all participants consisted of CHB patients. Patients' mean hepatic activity index (HAI) was 5.6 \pm 3.3 , and their mean fibrosis index was $2.2 \pm 2.0$.

\section{Quality of Life Scores}

The relationship between participants' SF-36 subscale scores and demographic characteristics was investigated. Men registered higher mean scores on all subscales, with physical functioning $(\mathrm{p}<0.001)$, social functioning $(\mathrm{p}=0.006)$, role physical $(\mathrm{p}<0.001)$, role emotional $(\mathrm{p}<0.001)$, vitality $(\mathrm{p}=0.024)$, and bodily pain $(\mathrm{p}=0.013)$ scores differing significantly from those of women. Significant differences were determined between education levels in terms of patients' SF-36 subscale scores. Significant differences in terms of physical functioning subscale scores were observed between primary school and university graduates, middle school and high school graduates, and middle school and university graduates ( $\mathrm{p}=0.008, \mathrm{p}=0.002$, and $\mathrm{p}<0.001$, respectively), while social functioning scores differed significantly between 
middle school and university graduates $(\mathrm{p}<0.001)$. Role physical, role emotional, and general health subscale score distributions differed significantly between middle school and university graduates $(\mathrm{p}<0.001)$, and bodily pain subscale score distributions differed significantly between primary school and university graduates $(\mathrm{p}=0.006)$.

Mean scores on all SF-36 subscales were higher among unmarried participants compared to married individuals, with social functioning, bodily pain, and general health subscale scores being significantly higher $(\mathrm{p}<0.001$, $\mathrm{p}=0.009$, and $\mathrm{p}=0.012$, respectively).

Significant differences were observed in SF-36 scores depending on patients' occupations. Physical functioning, social functioning, role emotional, and vitality subscale scores differed significantly between housewives and the unemployed $(p<0.001)$. Physical functioning, role physical, and role emotional scores also differed significantly between housewives and the self-employed $(\mathrm{p}<0.001)$. Score distributions among housewives and clerical workers differed significantly in the physical functioning, social functioning, and role physical subscales $(\mathrm{p}<0.001, \mathrm{p}=0.004$, and $\mathrm{p}=0.003$, relatively).

Mental health subscale scores differed significantly depending on smoking status $(\mathrm{p}=0.015)$. With the exception of role physical and role emotional, all mean SF-36 subscale scores were higher among patients who did not consume alcohol than among alcohol users, although statistical analysis could not be applied due to the insufficient number of alcohol users.

Physical functioning, role physical, role emotional, and vitality subscale scores differed significantly between participants living in urban areas and those living in outlying rural districts and villages $(\mathrm{p}<0.01)$. No significant difference was observed in participants' SF36 subscale scores depending on presence or absence of additional disease $(\mathrm{p}>0.05)$. Patients' SF-36 subscale score distributions according to sociodemographic variables are shown in Table 2.
Correlations were investigated between a number of sociodemographic and clinical characteristics and SF36 subscale scores. Interestingly, mental health subscale scores exhibited significant negative correlation only with duration of disease $(\mathrm{r}=-0.211, \mathrm{p}=0.002)$. Correlations between SF-36 subscale scores and age, education level, number of children, duration of disease, HAI, and fibrosis are summarized in Table 3.

\section{DISCUSSION}

Patients with chronic liver disease have lower HRQoL than the general population (10). When the relationship between demographic, clinical characteristics and quality of life and fatigue was evaluated in CHD patients, it was seen that age, gender, education, employment, comorbidity and disease stage affect fatigue and quality of life (11). In a study examining both general and disease-specific measures of HRQOL in Chinese patients, lower scores were reported in CHB patients compared to controls, especially in patients with cirrhosis (12). Nucleos(t)ide analogues used in treatment are powerful inhibitors of HBV replication, but long-term use affects patients' quality of life (13). Patients' sociodemographic characteristics and quality of life must be considered before starting long-term treatment. The present study evaluated the relationship between quality of life scores and sociodemographic characteristics in the $\mathrm{CHB}$ patients receiving antiviral therapy.

Numerous studies have reported impairment of HRQoL in patients with CHB. In a study a study evaluating the quality of life in patients with chronic viral hepatitis, it was reported that the quality of life of patients with $\mathrm{CHB}$ was lower than that of other chronic liver problems (14). A separate study revealed comparable HRQoL between inactive $\mathrm{HBV}$ carriers and healthy controls, but that this worsened as liver disease progressed. Significant impairment of HRQoL occurs in patients with $\mathrm{CHB}$ (15). The HRQoL of CHB patients can vary depending on their sociodemographic characteristics. Significantly lower HRQoL has been reported in women, unmarried patients, and individuals with chronic disease (6). In

\begin{tabular}{|lccccccc|}
\hline \multicolumn{6}{l}{ Table 3. Correlations netween SF-36 scores and various sociodemograhic variables and clinical characteristics $(\mathrm{r} / \mathrm{p})$} \\
\hline Variables & Age & Education level & Number of children & Duration of disease & HAI $^{*}$ & Fibrosis \\
\hline Physical functioning & $-0.313 /<0.001$ & $0.332 /<0.001$ & $-0.401 / 0.006$ & $-0.128 / 0.061$ & $-0.194 / 0.031$ & $-0.190 / 0.035$ \\
Social functioning & $-0.307 /<0.001$ & $0.238 /<0.001$ & $-0.072 / 0.640$ & $-0.306 /<0.001$ & $-0.122 / 0.178$ & $-0.181 / 0.044$ \\
Role physical & $-0.190 / 0.005$ & $0.316 /<0.001$ & $-0.234 / 0.121$ & $-0.037 / 0.585$ & $-0.076 / 0.402$ & $-0.102 / 0.258$ \\
Role emotional & $-0.188 / 0.006$ & $0.317 /<0.001$ & $-0.234 / 0.121$ & $-0.046 / 0.501$ & $-0.108 / 0.234$ & $-0.108 / 0.231$ \\
Mental health & $-0.090 / 0.189$ & $0.122 / 0.075$ & $-0.022 / 0.886$ & $-0.211 / 0.002$ & $-0.001 / 0.992$ & $-0.042 / 0.645$ \\
Vitality & $-0.171 / 0.012$ & $0.172 / 0.012$ & $-0.198 / 0.191$ & $-0.125 / 0.067$ & $0.044 / 0.628$ & $-0.059 / 0.517$ \\
Bodily pain & $-0.184 / 0.007$ & $0.200 / 0.003$ & $-0.193 / 0.204$ & $-0.157 / 0.022$ & $-0.139 / 0.124$ & $-0.243 / 0.006$ \\
General health & $-0.183 / 0.007$ & $0.250 /<0.001$ & $0.044 / 0.776$ & $-0.192 / 0.005$ & $-0.011 / 0.905$ & $-0.099 / 0.276$ \\
\hline
\end{tabular}

$\mathrm{r} / \mathrm{p}$ : Correlation coefficient/p value ${ }^{*}$ Hepatic Activity Index 


\begin{tabular}{|c|c|c|c|c|c|c|c|c|}
\hline Variables & $\begin{array}{c}\text { Physical } \\
\text { Functioning }\end{array}$ & $\begin{array}{c}\text { Social } \\
\text { Functioning } \\
\end{array}$ & $\begin{array}{c}\text { Role } \\
\text { Physical }\end{array}$ & $\begin{array}{c}\text { Social } \\
\text { Functioning }\end{array}$ & $\begin{array}{l}\text { Mental } \\
\text { Health }\end{array}$ & Vitality & $\begin{array}{l}\text { Bodily } \\
\text { Pain }\end{array}$ & $\begin{array}{c}\text { General } \\
\text { Health }\end{array}$ \\
\hline Sex & $\mathrm{p}<0.001$ & $\mathrm{p}=0.006$ & $\mathrm{p}<0.001$ & $\mathrm{p}<0.001$ & $\mathrm{p}=0.936$ & $\mathrm{p}=0.024$ & $\mathrm{p}=0.013$ & $\mathrm{p}=0.090$ \\
\hline Male & $\begin{array}{c}100.0 \\
(90.0-100.0)\end{array}$ & $\begin{array}{c}77.8 \\
(55.6-100.0)\end{array}$ & $\begin{array}{c}100.0 \\
(100.0-100.0)\end{array}$ & $\begin{array}{c}100.0 \\
(100.0-100.0)\end{array}$ & $\begin{array}{c}60.0 \\
(52.0-64.0)\end{array}$ & $\begin{array}{c}50.0 \\
(45.0-55.0)\end{array}$ & $\begin{array}{c}44.4 \\
(44.4-66.7)\end{array}$ & $\begin{array}{c}52.0 \\
(50.0-62.0)\end{array}$ \\
\hline Female & $\begin{array}{c}75.0 \\
(55.0-100.0)\end{array}$ & $\begin{array}{c}55.6 \\
(55.6-100.0)\end{array}$ & $\begin{array}{c}100.0 \\
(0.0-100.0)\end{array}$ & $\begin{array}{c}100.0 \\
(0.0-100.0)\end{array}$ & $\begin{array}{c}56.0 \\
(52.0-64.0)\end{array}$ & $\begin{array}{c}50.0 \\
(40.0-50.0)\end{array}$ & $\begin{array}{c}44.4 \\
(44.4-60.8)\end{array}$ & $\begin{array}{c}50.0 \\
(50.0-62.0)\end{array}$ \\
\hline Education level & $\mathrm{p}<0.001$ & $\mathrm{p}=0.003$ & $\mathrm{p}<0.001$ & $\mathrm{p}<0.001$ & $\mathrm{p}=0.106$ & $\mathrm{p}=0.087$ & $\mathrm{p}=0.018$ & $\mathrm{p}=0.002$ \\
\hline Primary school & $\begin{array}{c}75.0 \\
(40.0-100.0) \mathrm{a}\end{array}$ & $\begin{array}{c}77.8 \\
(55.6-90.0)\end{array}$ & $\begin{array}{c}100.0 \\
(0.0-100.0)\end{array}$ & $\begin{array}{c}100.0 \\
(0.0-100.0)\end{array}$ & $\begin{array}{c}52.0 \\
(40.0-60.0)\end{array}$ & $\begin{array}{c}42.5 \\
(35.0-50.0)\end{array}$ & $\begin{array}{c}44.4 \\
(44.4-44.4) \mathrm{a}\end{array}$ & $\begin{array}{c}52.0 \\
(47.0-62.0)\end{array}$ \\
\hline Middle school & $\begin{array}{c}85.0 \\
(60.0-100.0) \\
\text { b, c }\end{array}$ & $\begin{array}{c}55.6 \\
(55.6-83.4)\end{array}$ & $\begin{array}{c}100.0 \\
(0.0-100.0) \mathrm{a}\end{array}$ & $\begin{array}{c}100.0 \\
(0.0-100.0) \mathrm{a}\end{array}$ & $\begin{array}{c}56.0 \\
(52.0-64.0)\end{array}$ & $\begin{array}{c}50.0 \\
(40.0-50.0)\end{array}$ & $\begin{array}{c}44.4 \\
(44.4-66.3)\end{array}$ & $\begin{array}{c}50.0 \\
(48.5-55.0) \mathrm{a}\end{array}$ \\
\hline High school & $\begin{array}{c}100.0 \\
(85.0-100.0) b\end{array}$ & $\begin{array}{c}72.2 \\
(55.6-100.0)\end{array}$ & $\begin{array}{c}100.0 \\
(75.0-100.0)\end{array}$ & $\begin{array}{c}100.0 \\
(66.7-100.0)\end{array}$ & $\begin{array}{c}56.0 \\
(52.0-64.0)\end{array}$ & $\begin{array}{c}50.0 \\
(40.0-52.5)\end{array}$ & $\begin{array}{c}44.4 \\
(44.4-66.7)\end{array}$ & $\begin{array}{c}52.0 \\
(50.0-66.0)\end{array}$ \\
\hline University & $\begin{array}{c}100.0 \\
(90.0-100.0) \\
\text { a, c }\end{array}$ & $\begin{array}{c}77.8 \\
(66.7-100.0)\end{array}$ & $\begin{array}{c}100.0 \\
(100.0-100.0) \mathrm{a}\end{array}$ & $\begin{array}{c}100.0 \\
(100.0-100.0) \mathrm{a}\end{array}$ & $\begin{array}{c}60.0 \\
(52.0-68.0)\end{array}$ & $\begin{array}{c}50.0 \\
(50.0-57.5)\end{array}$ & $\begin{array}{c}55.6 \\
(44.4-77.8) \mathrm{a}\end{array}$ & $\begin{array}{c}57.0 \\
(51.0-67.0) \mathrm{a}\end{array}$ \\
\hline Marital status & $\mathrm{p}=0.052$ & $\mathrm{p}<0.001$ & $\mathrm{p}=0.105$ & $\mathrm{p}=0.084$ & $\mathrm{p}=0.104$ & $\mathrm{p}=0.085$ & $\mathrm{p}=0.009$ & $\mathrm{p}=0.011$ \\
\hline Married & $\begin{array}{c}90.0 \\
(65.0-100.0)\end{array}$ & $\begin{array}{c}66.7 \\
(55.6-90.0)\end{array}$ & $\begin{array}{c}100 \\
(25.0-100.0)\end{array}$ & $\begin{array}{c}100.0 \\
(0.0-100.0)\end{array}$ & $\begin{array}{c}56.0 \\
(52.0-64.0)\end{array}$ & $\begin{array}{c}50.0 \\
(40.0-50.0)\end{array}$ & $\begin{array}{c}44.4 \\
(44.4-66.7)\end{array}$ & $\begin{array}{c}52.0 \\
(50.0-60.0)\end{array}$ \\
\hline Unmarried & $\begin{array}{c}100.0 \\
(90.0-100.0)\end{array}$ & $\begin{array}{c}100.0 \\
(77.8-100.0)\end{array}$ & $\begin{array}{c}100.0 \\
(100.0-100.0)\end{array}$ & $\begin{array}{c}100.0 \\
(100.0-100.0)\end{array}$ & $\begin{array}{c}60.0 \\
(52.0-68.0)\end{array}$ & $\begin{array}{c}50.0 \\
(45.0-65.0)\end{array}$ & $\begin{array}{c}66.7 \\
(44.4-88.9)\end{array}$ & $\begin{array}{c}62.0 \\
(50.0-75.0)\end{array}$ \\
\hline Occupation & $\mathrm{p}<0.001$ & $\mathrm{p}=0.002$ & $\mathrm{p}<0.001$ & $\mathrm{p}<0.001$ & $\mathrm{p}=0.611$ & $\mathrm{p}=0.029$ & $\mathrm{p}=0.065$ & $\mathrm{p}=0.040$ \\
\hline Unemployed & $\begin{array}{c}100.0 \\
(85.0-100.0) \mathrm{a}\end{array}$ & $\begin{array}{c}77.8 \\
(55.6-100.0) a\end{array}$ & $\begin{array}{c}100.0 \\
(100.0-100.0) \mathrm{a}\end{array}$ & $\begin{array}{c}100.0 \\
(100.0-100.0) \mathrm{a}\end{array}$ & $\begin{array}{c}56.0 \\
(52.0-72.0)\end{array}$ & $\begin{array}{c}50.0 \\
(50.0-65.0) \mathrm{a}\end{array}$ & $\begin{array}{c}55.6 \\
(44.4-77.8)\end{array}$ & $\begin{array}{c}52.0 \\
(50.0-72.0)\end{array}$ \\
\hline Self-employed & $\begin{array}{c}70.0 \\
(55.0-100.0) \mathrm{b}\end{array}$ & $\begin{array}{c}55.6 \\
(55.6-77.8) \mathrm{b}\end{array}$ & $\begin{array}{c}100.0 \\
(0.0-100.0)\end{array}$ & $\begin{array}{c}100.0 \\
(0.0-100.0) \mathrm{b}\end{array}$ & $\begin{array}{c}56.0 \\
(52.0-64.0)\end{array}$ & $\begin{array}{c}50.0 \\
(40.0-50.0)\end{array}$ & $\begin{array}{c}44.4 \\
(44.4-55.6)\end{array}$ & $\begin{array}{c}50.0 \\
(47.0-55.0)\end{array}$ \\
\hline Clerical & $\begin{array}{c}100.0 \\
(85.0-100.0) \mathrm{c}\end{array}$ & $\begin{array}{c}77.8 \\
(55.6-77.8) \mathrm{c}\end{array}$ & $\begin{array}{c}100.0 \\
(100.0-100.0) b\end{array}$ & $\begin{array}{c}100.0 \\
(100.0-100.0)\end{array}$ & $\begin{array}{c}60.0 \\
(52.0-64.0)\end{array}$ & $\begin{array}{c}50.0 \\
(50.0-50.0)\end{array}$ & $\begin{array}{c}44.4 \\
(44.4-66.7)\end{array}$ & $\begin{array}{c}57.0 \\
(50.0-62.0)\end{array}$ \\
\hline Housewife & $\begin{array}{c}100.0 \\
(90.0-100.0) \\
\text { a, b, c }\end{array}$ & $\begin{array}{c}77.8 \\
(55.6-100.0) \\
a, b, c\end{array}$ & $\begin{array}{c}100.0 \\
(100.0-100.0) \\
\text { a,b }\end{array}$ & $\begin{array}{c}100.0 \\
(100.0-100.0) \\
\text { a,b }\end{array}$ & $\begin{array}{c}60.0 \\
(52.0-64.0)\end{array}$ & $\begin{array}{c}50.0 \\
(45.0-50.0) \mathrm{a}\end{array}$ & $\begin{array}{c}44.4 \\
(44.4-77.8)\end{array}$ & $\begin{array}{c}52.0 \\
(50.0-62.0)\end{array}$ \\
\hline Student & $\begin{array}{c}97.5 \\
(90.0-100.0)\end{array}$ & $\begin{array}{c}94.4 \\
(77.8-100.0)\end{array}$ & $\begin{array}{c}100.0 \\
(100.0-100.0)\end{array}$ & $\begin{array}{c}100.0 \\
(100.0-100.0)\end{array}$ & $\begin{array}{c}52.0 \\
(48.0-64.0)\end{array}$ & $\begin{array}{c}42.5 \\
(30.0-60.0)\end{array}$ & $\begin{array}{c}44.4 \\
(44.4-100.0)\end{array}$ & $\begin{array}{c}59.5 \\
(47.0-75.0)\end{array}$ \\
\hline Smoker & $\mathrm{p}=0.886$ & $\mathrm{p}=0.130$ & $\mathrm{p}=0.564$ & $\mathrm{p}=0.578$ & $\mathrm{p}=0.015$ & $\mathrm{p}=0.695$ & $\mathrm{p}=0.648$ & $\mathrm{p}=0.799$ \\
\hline No & $\begin{array}{c}92.5 \\
(65.0-100.0)\end{array}$ & $\begin{array}{c}77.8 \\
(55.6-100.0)\end{array}$ & $\begin{array}{c}100.0 \\
(25.0-100.0)\end{array}$ & $\begin{array}{c}100.0 \\
(33.3-100.0)\end{array}$ & $\begin{array}{c}60.0 \\
(52.0-64.0)\end{array}$ & $\begin{array}{c}50.0 \\
(40.0-55.0)\end{array}$ & $\begin{array}{c}44.4 \\
(44.4-66.7)\end{array}$ & $\begin{array}{c}52.0 \\
(50.0-62.0)\end{array}$ \\
\hline Yes & $\begin{array}{c}90.0 \\
(70.0-100.0)\end{array}$ & $\begin{array}{c}61.1 \\
(55.6-77.8)\end{array}$ & $\begin{array}{c}100.0 \\
(100.0-100.0)\end{array}$ & $\begin{array}{c}100.0 \\
(100.0-100.0)\end{array}$ & $\begin{array}{c}52.0 \\
(52.0-60.0)\end{array}$ & $\begin{array}{c}50.0 \\
(40.0-50.0)\end{array}$ & $\begin{array}{c}44.4 \\
(44.4-66.7)\end{array}$ & $\begin{array}{c}52.0 \\
(50.0-62.0)\end{array}$ \\
\hline \multicolumn{9}{|l|}{ Alcohol use } \\
\hline No & $\begin{array}{c}90.0 \\
(65.0-100.0)\end{array}$ & $\begin{array}{c}77.8 \\
(55.6-100.0)\end{array}$ & $\begin{array}{c}100.0 \\
(25.0-100.0)\end{array}$ & $\begin{array}{c}100.0 \\
(33.3-100.0)\end{array}$ & $\begin{array}{c}56.0 \\
(52.0-64.0)\end{array}$ & $\begin{array}{c}50.0 \\
(40.0-55.0)\end{array}$ & $\begin{array}{c}44.4 \\
(44.4-66.7)\end{array}$ & $\begin{array}{c}52.0 \\
(50.0-62.0)\end{array}$ \\
\hline Yes & $\begin{array}{c}87.5 \\
(60.0-100.0)\end{array}$ & $\begin{array}{c}50.0 \\
(44.4-61.1)\end{array}$ & $\begin{array}{c}100.0 \\
(100.0-100.0)\end{array}$ & $\begin{array}{c}100.0 \\
(100.0-100.0)\end{array}$ & $\begin{array}{c}56.0 \\
(52.0-60.0)\end{array}$ & $\begin{array}{c}50.0 \\
(42.5-50.0)\end{array}$ & $\begin{array}{c}44.4 \\
(44.4-66.7)\end{array}$ & $\begin{array}{c}51.0 \\
(50.0-52.0)\end{array}$ \\
\hline $\begin{array}{l}\text { Place of } \\
\text { residence }\end{array}$ & $\mathrm{p}=0.013$ & $\mathrm{p}=0.261$ & $\mathrm{p}=0.015$ & $\mathrm{p}=0.024$ & $\mathrm{p}=0.418$ & $\mathrm{p}=0.027$ & $\mathrm{p}=0.104$ & $\mathrm{p}=0.536$ \\
\hline Urban center & $\begin{array}{c}95.0 \\
(70.0-100.0) a\end{array}$ & $\begin{array}{c}77.7 \\
(55.5-100.0)\end{array}$ & $\begin{array}{c}100.0 \\
(50.0-100.0) \mathrm{a}\end{array}$ & $\begin{array}{c}100.0 \\
(54.1-100.0) \mathrm{a}\end{array}$ & $\begin{array}{c}58.0 \\
(52.0-64.0)\end{array}$ & $\begin{array}{c}50.0 \\
(40.0-55.0) a\end{array}$ & $\begin{array}{c}44.4 \\
(44.4-66.6)\end{array}$ & $\begin{array}{c}52.0 \\
(50.0-62.0)\end{array}$ \\
\hline Outlying district & $\begin{array}{c}90.0 \\
(70.0-100.0) \mathrm{b}\end{array}$ & $\begin{array}{c}77.7 \\
(55.5-100.0)\end{array}$ & $\begin{array}{c}100.0 \\
(100.0-100.0) \mathrm{b}\end{array}$ & $\begin{array}{c}100.0 \\
(83.3-100.0) b\end{array}$ & $\begin{array}{c}60.0 \\
(52.0-64.0)\end{array}$ & $\begin{array}{c}50.0 \\
(42.5-55.0) \mathrm{b}\end{array}$ & $\begin{array}{c}44.4 \\
(44.4-66.6)\end{array}$ & $\begin{array}{c}52.0 \\
(50.0-62.0)\end{array}$ \\
\hline Village & $\begin{array}{c}60.0 \\
(55.0-87.5) a, b\end{array}$ & $\begin{array}{c}66.6 \\
(55.5-77.7)\end{array}$ & $\begin{array}{c}0.0 \\
(0.0-100.0) a, b\end{array}$ & $\begin{array}{c}0.0 \\
(0.0-100.0) a, b\end{array}$ & $\begin{array}{c}56.0 \\
(50.0-60.0)\end{array}$ & $\begin{array}{c}40.0 \\
(25.0-50.0) \\
a, b\end{array}$ & $\begin{array}{c}44.4 \\
(38.8-44.4)\end{array}$ & $\begin{array}{c}50.0 \\
(845.0-61.0)\end{array}$ \\
\hline $\begin{array}{l}\text { Additional } \\
\text { disease }\end{array}$ & $\mathrm{p}=0.146$ & $\mathrm{p}=0.119$ & $\mathrm{p}=0.735$ & $\mathrm{p}=0.613$ & $\mathrm{p}=0.217$ & $\mathrm{p}=0.844$ & $\mathrm{p}=0.650$ & $\mathrm{p}=0.684$ \\
\hline No & $\begin{array}{c}95.0 \\
(67.5-100.0)\end{array}$ & $\begin{array}{c}77.8 \\
(55.6-100.0)\end{array}$ & $\begin{array}{c}100.0 \\
(38.0-100.0)\end{array}$ & $\begin{array}{c}100.0 \\
(41.7-100.0)\end{array}$ & $\begin{array}{c}58.0 \\
(52.0-64.0)\end{array}$ & $\begin{array}{c}50.0 \\
(40.0-55.0)\end{array}$ & $\begin{array}{c}44.4 \\
(44.4-66.7)\end{array}$ & $\begin{array}{c}52.0 \\
(50.0-62.0)\end{array}$ \\
\hline Yes & $\begin{array}{c}90.0 \\
(60.0-100.0)\end{array}$ & $\begin{array}{c}61.1 \\
(55.6-77.8)\end{array}$ & $\begin{array}{c}100.0 \\
(25.0-100.0)\end{array}$ & $\begin{array}{c}100.0 \\
(0.0-100.0)\end{array}$ & $\begin{array}{c}54.0 \\
(52.0-60.0)\end{array}$ & $\begin{array}{c}50.0 \\
(45.0-50.0)\end{array}$ & $\begin{array}{c}44.4 \\
(44.4-55.6)\end{array}$ & $\begin{array}{c}52.0 \\
(50.0-57.0)\end{array}$ \\
\hline
\end{tabular}


addition, studies employed different quality of life scales have observed that education level, age, duration of disease, and antiviral therapy affect HRQoL (16).

In a study evaluating fatigue-related risk factors in chronic hepatitis B patients, it was shown that male patients were significantly lower than female patients in terms of physical fatigue, mental fatigue, and decreased motivation. The study emphasizes that there is a negative correlation between fatigue and quality of life (17). One multicenter study from Turkey reported significantly lower HRQoL scores in women and unmarried patients (6). In contrast, in the present study, HRQoL was significantly lower in women and married patients. The difference in HRQoL scores between the sexes may be attributed to factors such as women enjoying less social support, generally later access to medical care than men, to their returning to work early before they have entirely recovered, and to their assumption of responsibilities (18). Since being married increases social and individual responsibilities, it can also adversely impact on HRQoL. However, it can also provide a healthier life by providing economic and social support $(19,20)$. There are striking differences between studies investigating the effect of marital status on HRQoL scores, and there are also studies reporting lower scores among unmarried patients (6).

Many studies have evaluated the effect of education level on quality of life in patients with chronic hepatitis $B(6,13)$. One study comparing inactive hepatitis B patients, $\mathrm{CHB}$ patients, and a healthy population in terms of quality of life reported significant correlations between education level and role limitations and bodily pain associated with physical health problems (21). A multicenter study from Turkey showed statistically significant correlations between all quality of life component scores and education level (6). In the present study, SF-36 subscale score distributions differed significantly depending on CHB patients' education levels, and positive correlation was determined between patient education level and quality of life subscale scores.

Individuals' occupations are another factor affecting quality of life. One study assessing quality of life in $\mathrm{CHB}$ patients reported low physical functioning, role physical, general health, and role emotional scores in patients with CHB (22). Similarly, in the present study, quality of life scores varied significantly depending on patients' occupational groups.

Mental health scores in the present study were lower among patients who smoked than among non-smokers. Lam et al. (23) reported higher physical component scores among smoker $\mathrm{CHB}$ patients.

Individuals living in urban centers may have better social communication and lifestyles. Analysis of
HRQoL in CHB patients in terms of place of residence in the present study revealed significant differences in physical functioning, role physical, role emotional, and vitality scores between those living in urban areas those resident in outlying districts and villages. However, one study of quality of life in patients with chronic hepatitis that place of residence had no effect on patients' quality of life (24).

No significant difference was also observed in the present study in any SF-36 subscale in terms of presence of additional disease. In contrast, however, some studies have reported lower quality of life scores in CHB patients with different chronic diseases than in those with no chronic disease (6).

Karacaer et al. (6) reported negative correlation between patient age and physical functioning subscale scores, and positive correlations between patient age and role emotional, mental health, and social functioning. In the present study, patient age was negatively correlated with physical functioning, social functioning, role physical, role emotional, vitality, bodily pain, and general health. Age-related decreases in numerous physical and mental abilities also result in a decrease in individuals' quality of life.

Negative correlation was detected in this study between duration of disease and mental and general health. Negative correlation was also detected between patients' HAI and physical functioning, and between fibrosis and physical functioning, social functioning, and bodily pain. However, one multi-center study reported no association between diagnosis of disease and duration of treatment and SF-36 scores. In addition, that study reported that since elevated alanine aminotransferase levels reduced quality of life scores, HBV DNA levels adversely affected subscales with the exception of vitality and physical and mental component scores (6).

\section{Limitations}

Significant increases have been reported in the physical components of quality of life, including physical functioning, role physical, bodily pain, and general health, following antiviral therapy (11). The present study analyzed quality of life of patients receiving antiviral therapy only in terms of sociodemographic characteristics. Comparative studies with patient groups not receiving antiviral therapy or inactive carriers might provide more extensive information about the effect of the treatment on quality of life. In addition, since a large proportion of the patients in this study were unwilling to disclose their monthly incomes, we were unable to assess the effect of economic status on quality of life. 


\section{CONCLUSION}

The quality of life of the CHB patients in the study group varied depending on demographic characteristics. Quality of life is dependent on individual factors, and the course and duration of the disease. Knowing that factors such as age, sex, education, place of residence, and duration of disease affects quality of life in CHB patients, assessing quality of life at controls, and providing psychiatric support when required, will be useful in increasing such patients' quality of life.

\section{ETHICAL DECLARATIONS}

Ethics Committee Approval: The study was approved by Atatürk University Faculty of Medicine Ethical Committee (Ethics approval certificate: B.30.2.ATA.0.01.00/95).

Informed Consent: All patients signed the free and informed consent form.

Referee Evaluation Process: Externally peer-reviewed.

Conflict of Interest Statement: The authors have no conflicts of interest to declare.

Financial Disclosure: The authors declared that this study has received no financial support.

Author Contributions: : Concept-HA, SY, MP, FKC, NP; Design - HA, SY, MP, FKC, NP; Supervision - HA, SY, MP, FKC, NP; Resources-HA, SY; Materials-HA, SY, FKC; Data Collection and/or Processing-HA, MP, NP; Analysis and/or Interpretation-HA, SY, NP; Literature Search-HA, SY; Writing Manuscript-HA, SY; Critical Review-HA, SY

\section{REFERENCES}

1. EASL. European Association for the Study of the Liver.Clinical Practice Guidelines on the management of hepatitis B virus infection. J Hepatol 2017; 67: 370-98.

2. Sarin SK, Kumar M, Lau GK, et al. Asian-Pacific clinical practice guidelines on the management of hepatitis B: a 2015 update. Hepatol Int 2016; 10: 1-98.

3. Terrault NA, Bzowej NH, Chang K-M, Hwang JP, Jonas MM, Murad MH. AASLD guidelines for treatment of chronic hepatitis B. Hepatology (Baltimore, Md) 2016; 63: 261.

4. Cai S, Yu T, Jiang Y, Zhang Y, Lv F, Peng J. Comparison of entecavir monotherapy and de novo lamivudine and adefovir combination therapy in HBeAg-positive chronic hepatitis B with high viral load: 48-week result. Clin Exp Med 2016; 16: 429-36.

5. CDC. Center for Disease Control and Prevention. HRQOL Concepts. Why is quality of life of important?April 01, 2016 2016 [Available from: http://www.cdc.gov/hrqol/concept.htm. updated: Accessed 26 February 2020.

6. Karacaer Z, Cakir B, Erdem H, et al. Quality of life and related factors among chronic hepatitis B-infected patients: a multicenter study, Turkey. Health Qual Life Outcomes 2016; 14: 153.

7. Ware Jr JE, Sherbourne CD. The MOS 36-item short-form health survey (SF-36): I. Conceptual framework and item selection. Med Care 1992: 473-83.

8. Pinar R. A new concept in health research: quality of life, testing the validity and reliability of a quality of life scale in chronic patients. Nurs Bull 1995; 9: 85-95.
9. Kocyigit H. Reliability and validity of the Turkish version of short form-36 (SF-36): a study in a group of patients will rheumatic diseases. Turk J Drugs Ther 1999; 12: 102-6.

10. Pradhan RR, Kafle Bhandari B, Pathak R, et al. The Assessment of Health-Related Quality of Life in Patients With Chronic Liver Disease: A Single-Center Study. Cureus 2020; 12: e10727.

11. Saffari M, Pakpour AH, Al Zaben F, Koening HG. Is there an association between Health Related Quality of Life, sociodemographic status and Fatigue in Patients with Chronic Hepatitis B? Acta Gastro-Enterologica Belgica. 2017; 80: 229-36.

12. Zhuang G, Zhang M, Liu Y, et al. Significant impairment of healthrelated quality of life in mainland Chinese patients with chronic hepatitis B : a cross-sectional survey with pair-matched healthy controls. Health and quality of life outcomes. 2014., 12 : 101.

13. Xue X, Cai S, Ou H, Zheng C, Wu X. Health-related quality of life in patients with chronic hepatitis B during antiviral treatment and off-treatment. Patient Prefer Adherence 2017; 11: 85-93.

14. Fotos NV, Elefsiniotis I, Patelarou A, et al. Psychological Disorders and Quality of Life Among Patients With Chronic Viral Hepatitis: A Single-Center Cross-Sectional Study With Pair-Matched Healthy Controls. Gastroenterol Nurs 2018; 41: 206-18.

15. Li L, Mo F, Hui EP, et al. The association of liver function and quality of life of patients with liver cancer. BMC Gastroenterol 2019; 19: 66.

16. Li G, Wang G, Hsu FC, et al. Effects of Depression, anxiety, stigma, and disclosure on health-related quality of life among chronic hepatitis b patients in Dalian, China. Am J Trop Med Hyg 2020; 102: 988-94.

17. Zhong C, Yin J, Zheng Z, Cai S, Gao Y. Risk factors associated with fatigue in chronic hepatitis $B$ patients. Patient Prefer Adherence 2019; 13: 1065-72.

18. Varì R, Scazzocchio B, D’Amore A, Giovannini C, Gessani S, Masella R. Gender-related differences in lifestyle may affect health status. Ann Ist Super Sanita 2016; 52: 158-66.

19. Monin JK. Commentary on "Low Mood and Risk of Dementia: The Role of Marital Status and Living Situation". Am J Geriatr Psychiatry. 2020; 28: 45-7.

20. Bulloch AGM, Williams JVA, Lavorato DH, Patten SB. The depression and marital status relationship is modified by both age and gender. J Affect Disord 2017; 223: 65-8.

21. Taşbakan MI, Sertöz ÖÖ, Pullukçu H, Çalik Ş, Sipahi OR, Yamazhan T. Comparison of quality of life in hepatitis B virus carriers versus chronic hepatitis B virus carriers versus the normal population. Turk J Med Sci 2010; 40: 575-83.

22. Özkan M, Çorapçioglu A, Balcioglu I, et al. Psychiatric morbidity and its effect on the quality of life of patients with chronic hepatitis B and hepatitis C. Int J Psychiatr Med 2006; 36: 283-97.

23. Lam ET, Lam CL, Lai C, Yuen M, Fong DY, So TM. Healthrelated quality of life of Southern Chinese with chronic hepatitis B infection. Health Qual Life Outcomes 2009; 7: 52.

24. Alay H, Özden K, Serpil E, Celik N, Parlak E, Parlak M. Assessment of Quality of Life of Patients with Chronic Hepatitis $\mathrm{B}$ and $\mathrm{C}$ Treated with Pegylated Interferon-alpha. Viral Hepatitis J 2018; 24: 25. 